\title{
Research on the Characteristics of Turnout Flow Based on the Eccentricity Index of Maximum Flow Velocity
}

\author{
$\mathrm{W}$ XI ${ }^{\mathrm{a}}$ and $\mathrm{W} \mathrm{G} \mathrm{LU}{ }^{\mathrm{a}, 1}$ \\ ${ }^{a}$ College of Hydraulic Science and Engineering, Yangzhou University, Yangzhou, \\ 225009 China
}

\begin{abstract}
On the one hand, the characteristics of the turnout flow in the river are related to the stability of the turnout flow, and on the other hand, it is related to the safety of the flowing buildings near the bend turnout river. To observe the characteristics of the flow field in the chaotic river, the open channel surface layer digital particle image velocity (DPIV) system is used to measure the data in the physical model of the curved turnout river, and the maximum flow eccentricity (MFE) of the channel section is established. The flow pattern of the turnout flow is analyzed. The results show that the MFE index can effectively evaluate the flow pattern of the turnout flow. The MFE of the upper stream section of the curved river changes with the sudden change position of the flow between twice to 3.5 times the water surface width from the center point, and moves upstream with the increase of the flow rate, and moves downstream with the increase of the water level. This characteristic has guiding significance for the adjustment of the flow pattern of the front pool of the building.
\end{abstract}

Keywords. Curved turnout flow, maximum flow eccentricity, numerical simulation, physical model

\section{Introduction}

The centrifugal force, inertial force, superimposed vortex and separation phenomenon of the curved turnout flow lead to the change of the turbulent structure of the water flow, which makes the flow pattern of the fork flow more complicated. Turnout flow is widely used in water conservancy engineering and agricultural engineering. Many researchers have conducted a lot of research on the complicated inlet flow pattern of curved turnout flow: Luo [1], Kesserwani [2], Huang [3] studied the flow pattern changes of the open channel confluence under different confluence angles, they found that the larger the intersection angle, the more uneven the flow velocity distribution at the intersection; van Balen [4], Xu D [5] used model test and numerical simulation methods, analyze and study the water flow characteristics of the elbow under different working conditions; Zeng [6], Yuan [7] summarized the general laws of the inflow and outflow patterns through the study of the open channel hydrodynamic field; Wang Xi [8], Zeng J [9], Xu C D [10] used physical and numerical methods to analyze the

${ }^{1}$ W G LU, College of Hydraulic Science and Engineering, Yangzhou University, Yangzhou, 225009 China; E-mail: wglu@yzu.edu.cn. 
hydraulic characteristics in the flow channel of the pumping station; Sudo [11] and Ahmed [12] researched the turbulence characteristics and average velocity changes of the bends at different intersection angles; Weber [13] and Zaji A H [14] adopted a combination of physical model and numerical model to analyze and find that the crosssectional circulation can reflect the three-dimensional flow characteristics of the confluent flow under large flow; Heydari N [15] used SPIV to analyze the influence of the upstream river bank angle on turbulence characteristics and the potential coherent structure related to the development of local scour near the front edge of the longitudinal structure; Luo $\mathrm{H}$ [16] used numerical simulation methods to explore the flow velocity distribution and energy loss at the joint; Nazari-Sharabian M [17] used FLOW-3D software to analyze the flow topology at the junction of the open channel and the lateral drainage pipe. In summary, there is currently no research on the flow characteristics of the turnout flow based on the maximum velocity eccentricity index. This paper takes a turnout flow in an actual project as the research object to carry out a numerical simulation study. The Large Eddy Model (Large Eddy Model) is selected to close the time-average equation of two-phase flow, the semi-implicit SIMPLE (SemiImplicit Method for Pressure-Linked Equations) algorithm is used when solving the velocity and pressure coupling equations, and the VOF (Volume of Fluid) method is used to simulate the free surface, And the open channel surface layer digital particle image velocity (digital particle image velocity, DPIV) is used to collect the surface layer flow patterns under different working conditions in the produced test model, and the results of the numerical simulation are mutually corroborated. At the same time, the MFE index was established to analyze the flow characteristics of the curved turnout flow in several aspects such as flow process, flow rates, and water level, and provide some reference data for the flow pattern adjustment of the curved turnout flow.

\section{Methodology}

\subsection{Build Physical Models}

In order to study the surface flow pattern of the turnout flow, combined with actual engineering, a trapezoidal channel with a $90^{\circ}$ bend turnout river is used as a prototype, and the cross section of the river is trapezoidal. The upper and lower reaches of the bend turnout river are simulated to be $300 \mathrm{~m}$ in the upper and lower reaches. The channel width is $16 \mathrm{~m}$ and the depth is $3 \mathrm{~m}$. The water flows in from the A river, the main flow diverges into the $\mathrm{C}$ river through the bends and the other flows out through the $\mathrm{B}$ river.

According to the relevant hydraulic model test procedures requirements, a normal model with geometric scale $\lambda=10$ was established and verified. The entire test model includes a river model, overflow plate, water tank, pipeline, and auxiliary pump. The overall layout of the test model is shown in figure 1 and figure 2.

To obtain the flow pattern of the turnout flow, the surface layer flow field uses the DPTV surface flow field acquisition system to collect flow field data. The DPIV highdefinition flow field measurement system is a large-scale surface flow field measurement system based on particle image velocity measurement technology (Particle Image Velocity). The concrete river channel is made of PVC board to simulate the surface in contact with the water flow. During the test, the flow meter and valve are used to control the water flow of the model, and the downstream water outlet is used to 
adjust the water level by the stacked beam overflow plate. When the flow and water levels are stable, use DPIV to collect flow field data. Then use Tepolt post-processing software to analyze the surface flow field data, and obtain the corresponding flow field vector and cloud image.

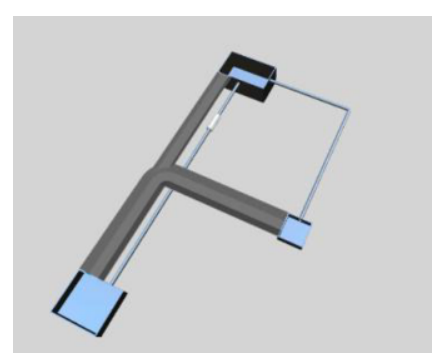

Figure 1. Schematic diagram of physical model test.

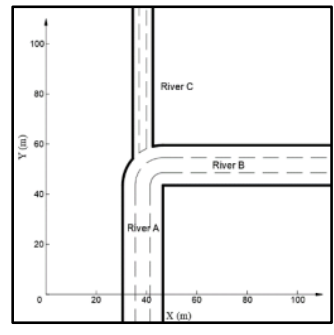

Figure 2. The model overall layout.

\subsection{Relevant Parameter}

\subsubsection{VOF Method}

The basic idea of the VOF method is: Define volume rate function $F=F(x, y, z, t)$ Indicates the relative ratio of the volume of fluid in the calculation area to the volume of the calculation area. For a certain computing unit, when $F(x, y, z, t)=1$, it means that the unit is full; when $F(x, y, z, t)=0$, it means that the unit is empty; when $0<F(x, y, z, t)<1$, it means that part of the unit is filled with liquid. Obviously, the free surface exists in the third unit. The gradient of $\mathrm{F}$ can be used to determine the normal direction of the free boundary. After calculating the value and gradient of each element, the approximate position of the free surface in each element can be determined. In each unit, the sum of the volume fractions of water and gas is 1. Compared with the real single-phase flow, there will be one more (water or gas) volume fraction variable. Let $a_{w}$ denote the volume fraction of water, then the volume fraction of gas $a_{a}$ can be expressed as: $a_{a}=1-a_{w}$.

As long as the volume fractions of water and gas are known everywhere in the flow field, the unknown quantities and characteristic parameters shared by all other water and gas can be represented by the weighted average of the volume integral. So in any given unit, these variables and characteristic parameters either represent pure water or gas, or a mixture of the two. The determination of the water-air interface requires solving the following equation:

$$
\frac{\partial a_{w}}{\partial t}+\bar{u}_{i} \frac{\partial a_{w}}{\partial x_{i}}=0
$$

$t$ is time. After the introduction of VOF, $\rho$ and $v$ are functions of the volume fraction, not constants, and through the iterative solution to the volume fraction of water $a_{w}, \rho$ and $v$ can be obtained by the following formula: 


$$
\begin{aligned}
& \rho=a_{w} \rho_{w}+\left(1-a_{w}\right) \rho_{a} \\
& v=a_{w} \rho_{w}+\left(1+a_{w}\right) v_{a}
\end{aligned}
$$

In the formula, $\rho_{a}$ and $\rho_{w}$ indicates the density of air and water respectively; $v_{a}$ and $v_{w}$ indicates the molecular viscosity coefficients of air and water respectively.

\subsubsection{Mesh Subdivision}

UG modeling software is used to establish the river channel geometric model. Divide the unstructured grid, combine actual engineering observations, select the flow field at the inlet water level $H$ of River A at $1.8 \mathrm{~m}, 2.1 \mathrm{~m}, 2.6 \mathrm{~m}, 2.8 \mathrm{~m}, 2.9 \mathrm{~m}, 3.0 \mathrm{~m}$ for the model establishment and grid division. Considering the influence of the number of grids on the accuracy of the numerical simulation, first explore the head loss of the grid in the calculation domain. The head loss calculation formula is as follows:

$$
h_{f}=\frac{\left(P_{\text {in }}-P_{\text {out }}\right)}{\rho g}
$$

$h_{f}$ is the head loss based on the average quality; $P_{i n}, P_{\text {out }}$ is the total pressure of the inlet section and outlet section; $\rho_{c}$ is the density of water at $4{ }^{\circ} \mathrm{C} ; g$ is the acceleration of gravity。

The results show that when all the water level errors are within $2 \%$ and the number of grids reaches 2896824, the water level error does not exceed $1 \%$. At this time, increasing the number of grids has little effect on the calculation results of the water level error. Considering calculation accuracy and calculation time, 2890000 grids are finally used for calculation. For each grid model, Simulate the inlet flow $Q$ for River A is $32 \mathrm{~m}^{3} \cdot \mathrm{s}^{-1}, 38 \mathrm{~m}^{3} \cdot \mathrm{s}^{-1}, 46 \mathrm{~m}^{3} \cdot \mathrm{s}^{-1}, 50 \mathrm{~m}^{3} \cdot \mathrm{s}^{-1}, 54 \mathrm{~m}^{3} \cdot \mathrm{s}^{-1}, 60 \mathrm{~m}^{3} \cdot \mathrm{s}^{-1}$.

Table 1. Different grid lines under the water level.

\begin{tabular}{lll}
\hline Total number of grids & $\mathbf{H} / \mathbf{m}$ & $\mathbf{Q} /\left(\mathbf{m}^{\mathbf{3}} \cdot \mathbf{s}^{-1}\right)$ \\
\hline 2258794 & 1.8 & 38 \\
2492631 & 2.1 & 38 \\
2896824 & 2.6 & 38 \\
3090634 & 2.8 & 38 \\
3272061 & 2.9 & 38 \\
3514412 & 3.0 & 38 \\
\hline
\end{tabular}

The calculation model uses the VOF model. The inlet is selected at the upper reaches of the A river, and the outlet is selected at the lower reaches of the $\mathrm{C}$ and $\mathrm{B}$ rivers. The solid wall in the flow area is constrained by the wall boundary condition, and the solid wall adopts the non-slip boundary condition, that is, the fluid is at a static state on the solid wall. The adaptive second-order upwind style is adopted, the effect of gravity is considered in the calculation process, and the PISO algorithm suitable for the calculation of unsteady compressible flow is adopted. 


\section{Simulation Analysis and Discussion}

\subsection{Analysis of Numerical Simulation Results}

\subsubsection{Results of Different Flow Rates}

The design uses a three-dimensional model of the river channel when the water level is $2.1 \mathrm{~m}$ to perform numerical simulation calculations to study the change of the eccentricity of the maximum flow velocity of the river under different flow rates. Here are the velocity cloud diagrams of the surface layer numerical calculation under three different flow rates, as shown in figure 3 .

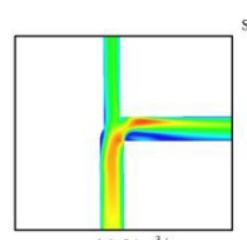

(a) $54 \mathrm{~m}^{3} / \mathrm{s}$

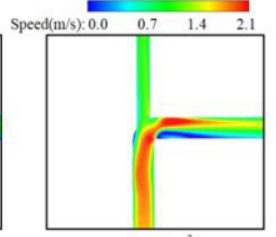

(b) $46 \mathrm{~m}^{3} / \mathrm{s}$

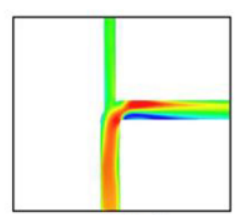

(c) $38 \mathrm{~m}^{3} / \mathrm{s}$

Figure 3. Flow rate cloud map.

As can be seen from the above figure, there is an obvious high-velocity area at the center of the inflow channel of River A. As the water level decreases, the range of the high-velocity area gradually decreases. The surface layer inflow of River A is relatively stable, and the flow velocity of the water entering the right side of the bend is relatively high. Large, diverging into the B river, the direction of the water flow is to the left, The high-speed area is concentrated on the left bank of the exit curve, and the speed is relatively high. There is a large low-velocity vortex at the exit of River B. As the water level decreases, the high-speed area tends to the shore, and the range of the lowvelocity vortex gradually decreases. Due to the change of the direction of the water entering the turnout river, the high-velocity area of the A River then bends along the direction of the water flow, and the bending point gradually moves downstream as the flow in the river increases.

\subsubsection{Results of Different Water Levels}

The design uses a three-dimensional model of the river channel with a flow rate of $38 \mathrm{~m}^{3} \cdot \mathrm{s}^{-1}$ for numerical simulation calculations to study the surface flow characteristics of the turnout flow under different water levels. Here are three numerically calculated velocity cloud diagrams at different water levels, as shown in the figure 4 below:

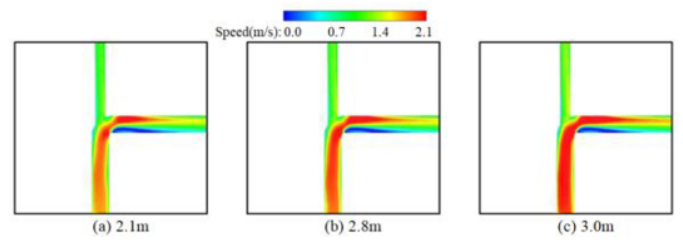

Figure 4. Flow rate cloud map. 
As can be seen from the above figure, there is an obvious high-velocity area at the center of the inflow channel in A river. As the water level decreases, the range of the high-velocity area gradually decreases. The surface flow of A river is relatively stable, and the water flow enters the right side of the bend with a higher velocity. After the diversion enters the $\mathrm{B}$ river, the direction of the water flow is biased to the left. The high-velocity area gathers on the left bank of the exit bend, and the flow velocity is higher. There is a large low-speed vortex on the side, and as the water level decreases, the high-velocity area tends to the shore, and the range of the low-speed vortex gradually decreases. The inflow of the A river along the direction of the water flow is on the right side of the center of the river channel, and the flow rate is large. Due to the change in the direction of the water entering turnout river, the high-velocity area of the A river bends along the direction of the water flow, and the bending point gradually moves upstream as the river increases.

\subsection{Analysis of Physical Model Flow Field}

Research on the flow field of curved turnouts, using DPIV to measure the flow field, and Tecplot to process the data. The physical model selects the two working conditions shown in figure 3(b) and figure 4(b) in the numerical simulation for verification. According to the collected data, the flow velocity cloud diagram and flow velocity vector diagram are shown in figure 5. According to the flow field obtained by analyzing the physical model test data, comparing the results of the numerical simulation with a water level of $2.1 \mathrm{~m}$ and a flow rate of $38 \mathrm{~m}^{3} / \mathrm{s}$, it can be seen that the size and location of the recirculation zone are basically the same, and the distribution of the high-velocity zone is basically the same, indicating the numerical simulation The results are basically consistent with the physical model test results, which shows that the numerical simulation results are credible.

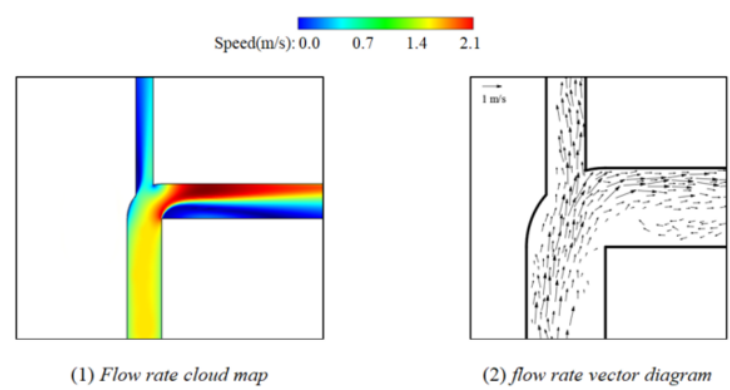

Figure 5. Results of DPIV.

\subsection{Maximum Flow Velocity Eccentricity Index}

To quantitatively compare and evaluate the flow pattern of the turnout flow, the MFE of the flow cross-section is established, which indicates the deviation degree of the maximum velocity from the center of the channel. The dimensionless number is based on the center of the velocity measurement section of the river channel, and the deviation degree of the peak velocity point appearing in the section velocity distribution graph relative to the base point is analyzed. The specific calculation formula is shown in formula (5). 


$$
F_{k}=\frac{\left|l_{p}-\frac{1}{2} X_{n}\right|}{\frac{1}{2} X_{n}}
$$

$F_{k}$ is the MFE corresponding to section $\mathrm{k}, l_{p}$ is the shortest distance between the peak flow rate and the river bank (If there are multiple peaks, take the weighted average), $X_{n}$ is the water surface width. Express the water surface width in terms of slope ratio $\tan \theta$, bottom width $X_{d}$, and water depth h, and simplify $F_{k}$ to:

$$
F_{k}=1-\frac{2 l_{p}}{2 h \tan \theta+X_{d}}
$$

Water is viscous. The flow at the edge of the river frictions with the river bank and is slowed down. The flow at the edge and the flow at the center of the river form a velocity gradient due to the viscosity, resulting in the fastest water velocity in the center of the normal river, and the closer the water is to the bank. The slower the flow rate. The eccentricity value of water flow is between 0 and 1 . The closer the value is to 1 , the greater the MFE is. And the high-velocity area deviates more from the center of the river channel, on the contrary, the smaller the MFE is, the smaller the high-velocity area deviates from the center of the river channel. Analyzing the flow pattern with the MFE can directly reflect the deviation of the high flow velocity area of each section from the center of the river channel so that the distribution trend of the flow velocity can be analyzed.

\subsubsection{Analyze Flow Patterns by $M F E$}

Set characteristic cross-sections at $15 \mathrm{~m}$ away from the bend in the $\mathrm{A}, \mathrm{B}$, and $\mathrm{C}$ rivers. The specific layout is shown in figure 6. Table 2 is the comparison table of the MFE of the three characteristic sections of the turnout flow. It can be seen from the table that the eccentricity of section a is 0.34 , the high-velocity area is close to the center of the river, and the surface flow regime is better. The eccentricity of section $b$ is as high as 0.73 , and the high-velocity area deviates greatly from the center of the channel section, indicating that the surface layer flow before entering the $\mathrm{C}$ river is turbulent, the flow of the gate hole is uneven, and the eccentricity of section $\mathrm{c}$ is 0.56 , and the highvelocity area is biased to the left The riverbank, scouring the river bank on the left side of the bifurcation.

Table 2. MFE of each section in different schemes.

\begin{tabular}{llll}
\hline & Section $\mathrm{a}$ & Section $\mathrm{b}$ & Section $\mathrm{c}$ \\
\hline MFE & 0.34 & 0.73 & 0.56 \\
\hline
\end{tabular}




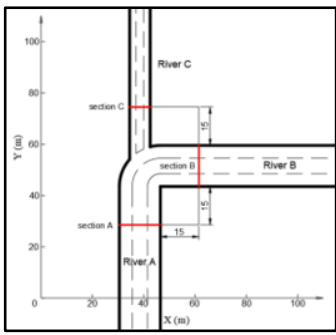

Figure 6. Schematic diagram of physical model test.

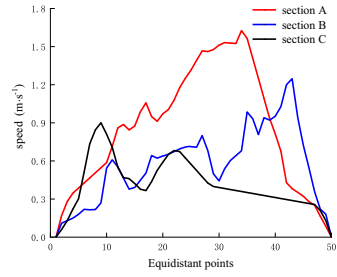

Figure 7. Flow velocity map of each section.

Combining the data collected by DPIV, using Tecplot software to process the flow velocity distribution diagrams of 3 characteristic sections, as shown in figure 7 . It can be seen from the figure that the flow velocity at section a of River A is relatively high, and the main flow of the surface layer flows along the right side of the center of the river, which is mainly affected by the shape of the turnout channel. The flow velocity at section $\mathrm{b}$ before entering river $\mathrm{C}$ fluctuates greatly, the surface velocity is unevenly distributed, and the flow velocity on the right side is relatively large. It is caused by the rapid change of the direction of water flow after the inertia hits the embankment on the right side of the turnout flow. and the main flow of the surface layer is biased to the left side of the channel center, and the water flow hits the left bank. This is mainly caused by the shape of the river channel and the inertia of the water flow. Combined with the analysis of the flow pattern, it is found that the flow pattern of section A is relatively good among these three sections, the MFE is small, while the flow patterns of sections $\mathrm{B}$ and $\mathrm{C}$ are poor, and the MFE is large. In summary, the flow regime is poor in places with large eccentricity, and the flow regime is relatively good in places with small eccentricity. It shows that the MFE can be used to judge the flow pattern.

\subsection{Features of MFE}

In order to quantitatively analyze the change of the MFE of the turnout flow with the flow process, according to the results of numerical simulation and physical model test, Suppose the center of the triangle determined by the intersection of the center lines of the three rivers of $\mathrm{A}, \mathrm{B}$, and $\mathrm{C}$ is the starting point of the center of the turnout flow (referred to as the "center point"), Establish 12 analysis sections. The starting section 1 is located at a distance of 2 times the average water surface width of River A from the center point, and the end section 12 is at a distance of 3.1 times the average water surface width of River A from the center point. The specific layout is shown in figure 8 . Each section is set with 50 equidistant measuring points to measure the surface flow velocity. 


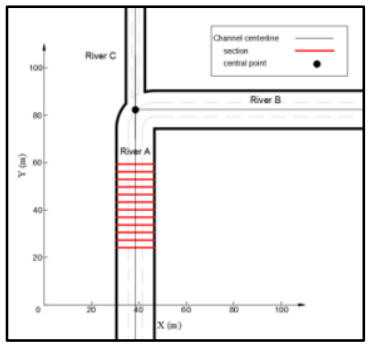

Figure 8. Diagram of the position.

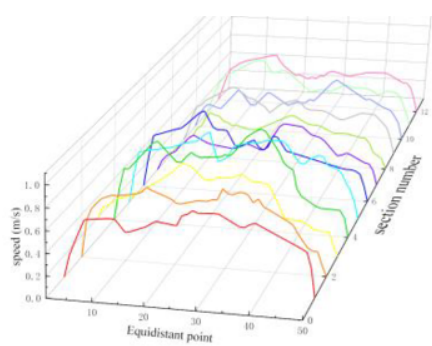

Figure 9. flow rate in different distance.

In order to quantitatively study the eccentricity of the maximum flow velocity in the main inflow channel A with the flow process change, combined with the actual engineering simulation, the water level is $2.1 \mathrm{~m}$ and the flow rate is $38 \mathrm{~m}^{3} \cdot \mathrm{s}^{-1}$. After collecting the flow field data by DPIV, Tecplot software was used to process the velocity distribution diagrams of 12 sections at different positions, as shown in figure 9 . Calculate the MFE of the 12 sections using the formula for calculating the maximum velocity eccentricity of the section, and draw the distribution of the MFE of the different sections along the flow process according to the data, as shown in figure 10 .

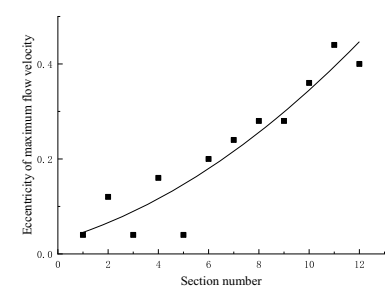

Figure 10. eccentricity at maximum flow rate in different section.

It can be seen from figures 9 and 10 that between 2.0 times the river width and 2.5 times the river width, the MFE fluctuates below 0.2 , the high-velocity area is stable in the middle of the river, and the river surface flow is good. Starting from 2.5 times the river width, the MFE began to rise sharply and showed a linear relationship. The highvelocity area began to shift to the center of the river channel, and the upstream river bank was scoured. It can be seen that, under this working condition, the point of change of the MFE of the inflow of the river A is at 2.5 times the average river width upstream of the center point.

\subsection{Impact of Water Level Changes}

In order to analyze the change of the MFE of the section under the same water level and different flow rates, the water level in the simulation project is selected as $2.1 \mathrm{~m}$, and the flow rate is $32 \mathrm{~m}^{3} \cdot \mathrm{s}^{-1}, 38 \mathrm{~m}^{3} \cdot \mathrm{s}^{-1}, 46 \mathrm{~m}^{3} \cdot \mathrm{s}^{-1}, 50 \mathrm{~m}^{3} \cdot \mathrm{s}^{-1}, 54 \mathrm{~m}^{3} \cdot \mathrm{s}^{-1}$ and $60 \mathrm{~m}^{3} \cdot \mathrm{s}^{-1}$, Taking MFE of River A as the research object, the variation analysis of MFE of the A river section under different flow rates is shown in figure 11 , In order to analyze the 
change of the MFE of the section under the same flow and different water levels, the flow rate of $38 \mathrm{~m}^{3} \cdot \mathrm{s}^{-1}$ in the simulation project is selected and the water level of River A is $1.8 \mathrm{~m}, 2.1 \mathrm{~m}, 2.6 \mathrm{~m}, 2.8 \mathrm{~m}, 2.9 \mathrm{~m}, 3.0 \mathrm{~m}$, The results are shown in figure 12 below.

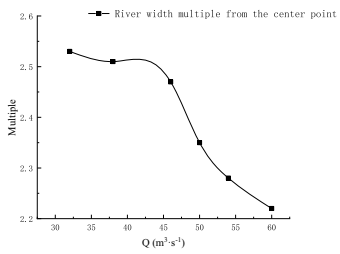

Figure 11. Inflection point of eccentricity at maximum flow rate under different flow.

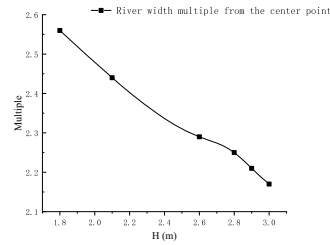

Figure 12. Inflection point of eccentricity at maximum flow rate under different water levels.

It can be seen from figure 11 that with the increase of the flow, the position where the MFE of the same channel changes continuously moves upstream. This is because the greater the flow, the greater the inertial force on the water flow, which will cause the high-velocity area to shift in advance, increase the area of bad flow in the river channel, increase the erosion of the riverbank, and increase the range of river regime changes. Therefore, during the flood season of the basin, it is not only necessary to supervise the turnout section of the bend, but also to inspect the river banks on both sides of the upstream.

It can be seen from figure 12 that the sudden change point of the MFE moves downstream with the decrease of the water level. This is because the lower the water level, the greater the flow velocity and the greater the inertial force of the water flow. Therefore, the position where the MFE changes suddenly.

\section{Conclusion}

The pool flow pattern of the turnout flow affects the safety of the river engineering. This study uses a combination of numerical simulation technology and physical model test and combined with MFE to analyze the flow characteristics of the turnout flow, and the following general conclusions were obtained:

(1) The MFE of the section can be used to intuitively evaluate the flow pattern of the turnout. The flow pattern of the river section with large eccentricity is poor, and the flow pattern of the section with small eccentricity is better.

(2) In the main inflow channel of the turnout flow, the MFE of the cross-section will change with the river regime along with the flow process.

(3) In the main inflow channel of the turnout flow, the position where the MFE of the cross-section has abrupt changes moves upstream with the increase of the overflow. The location of the maximum flow increases with the flow rate and gradually deviates from the center of the river channel The gradient of the water flow velocity becomes more and more obvious, which will scour the river slope and cause harm.

(4) The lower the water level, the greater the flow velocity, and the greater the inertial force of the flow in the main inflow channel of the turnout flow, the sudden change point of the MFE will move upstream as the water level rises. 


\section{Acknowledgments}

The work was supported by the Jiangsu Graduate Research and Innovation Program (KYCX20_2980), the National Natural Science Foundation of China (No. 51779215; No. 52079120) and the Priority Academic Program Development of Jiangsu Higher Education Institutions (PAPD).

\section{References}

[1] Luo H, Fytanidis DK, Schmidt AR \& García MH. Comparative 1D and 3D numerical investigation of open-channel junction flows and energy losses. Advances in Water Resources. 2018 May; 117: 120139.

[2] Kesserwani G, Ghostine R, Vazquez J, Mosé R, Abdallah M \& Ghenaim A. Simulation of subcritical flow at open-channel junction. Advances in Water Resources. 2007 Aug; 31(2): 287-297.

[3] Huang J, Weber LJ \& Lai YG. Three-dimensional numerical study of flows in open-channel junctions. Journal of Hydraulic Engineering. 2002 Mar; 128(3): 268-280.

[4] Van Balen W, Blanckaert K \& Uijttewaal WS. Analysis of the role of turbulence in curved openchannel flow at different water depths by means of experiments, LES and RANS. Journal of Turbulence. 2010 Apr; (11): N12.

[5] Xu D, Ji C, Bai Y \& Song X. Three-dimensional numerical investigation on the influence of geometric shape on flow in river bends. Journal of Hydroinformatics. 2017 Sep; 19(5): 666-685.

[6] Zeng C \& Li CW. A hybrid RANS-LES model for combining flows in open-channel T-junctions. Journal of Hydrodynamics. 2010 Oct; 22(1): 154-159.

[7] Yuan S, Tang H, Xiao Y, Qiu X, Zhang H \& Yu D. Turbulent flow structure at a 90-degree open channel confluence: Accounting for the distortion of the shear layer. Journal of Hydro-Environment Research. 2016 May; 12: 130-147.

[8] Xi W, Lu W, Wang C \& Xu B. Optimization of the hollow rectification sill in the forebay of the pump station based on the PSO-GP collaborative algorithm. Shock and Vibration. 2021 May.

[9] Zeng J, Constantinescu G, Blanckaert K, et al. Flow and bathymetry in sharp open-channel bends: Experiments and predictions. Water Resources Research. 2008 Sep; 44(9).

[10] Xu CD, Wang RR, Liu H, Zhang R, Wang MY \& Wang Y. Flow pattern and anti-silt measures of straight-edge forebay in large pump stations. International Journal of Heat and Technology. 2018 Apr; 36(3): 1130-1139.

[11] Sudo K, Sumida M \& Hibara H. Experimental investigation on turbulent flow in a circular-sectioned 90-degree bend. Experiments in Fluids. 1998 Jun; 25(1): 42-49.

[12] Ahmed F. Three-dimensional mean velocity analysis of a 30 degree bend flow. Journal of Engineering Mechanics. 2000 Dec; 126(12): 1262-1272.

[13] Weber LJ, Schumate ED \& Mawer N. Experiments on flow at a 90 open-channel junction. Journal of Hydraulic Engineering. 2001 May; 127(5): 340-350.

[14] Zaji AH \& Bonakdari H. Velocity field simulation of open-channel junction using artificial intelligence approaches. Iranian Journal of Science and Technology, Transactions of Civil Engineering. 2018 Oct; 43(1): 549-560.

[15] Heydari N, Diplas P, Nathan Kutz J \& Sadeghi ES. Modal analysis of turbulent flow near an inclined bank-longitudinal structure junction. Journal of Hydraulic Engineering. 2020 Dec; 147(3): 04020100.

[16] Luo H, Fytanidis DK, Schmidt AR \& García MH. Comparative 1D and 3D numerical investigation of open-channel junction flows and energy losses. Advances in Water Resources, 2018 May; 117: 120139.

[17] Nazari-Sharabian M, Karakouzian M \& Hayes D. Flow topology in the confluence of an open channel with lateral drainage pipe. Hydrology. 2020 Aug; 7(3): 57. 